\title{
Optimising Chlamydia Screening - a city and countywide approach in Nottinghamshire
}

\author{
Sally Handley ${ }^{*}$, Deborah Shaw², Carl Neal', Carol Ford', Nick Romilly', Jonathan Gribbin', \\ John Saunders ${ }^{2 * *}$, Ruth Taylor ${ }^{4}$, Elizabeth Carlin ${ }^{5}$
}

'Nottinghamshire County Council; ${ }^{2}$ National Chlamydia Screening Programme, Public Health England; ${ }^{3}$ Nottingham City Council; ${ }^{4}$ Nottingham University Hospitals NHS Trust; ${ }^{5}$ Sherwood Forest Hospitals NHS Foundation Trust

\section{Background}

\section{National Context:}

The English National Chlamydia Screening Programme (NCSP)' offers testing to sexually active young people under 25 years, as part of routine appointments in primary care, sexual health and other relevant settings.

The NCSP Chlamydia Care Pathway $(\mathrm{CCP})^{2}$ tool aims to identify areas across seven stages of case management where implementing change may lead to improved outcomes.

Figure I:The Chlamydia Care Pathway ${ }^{2}$

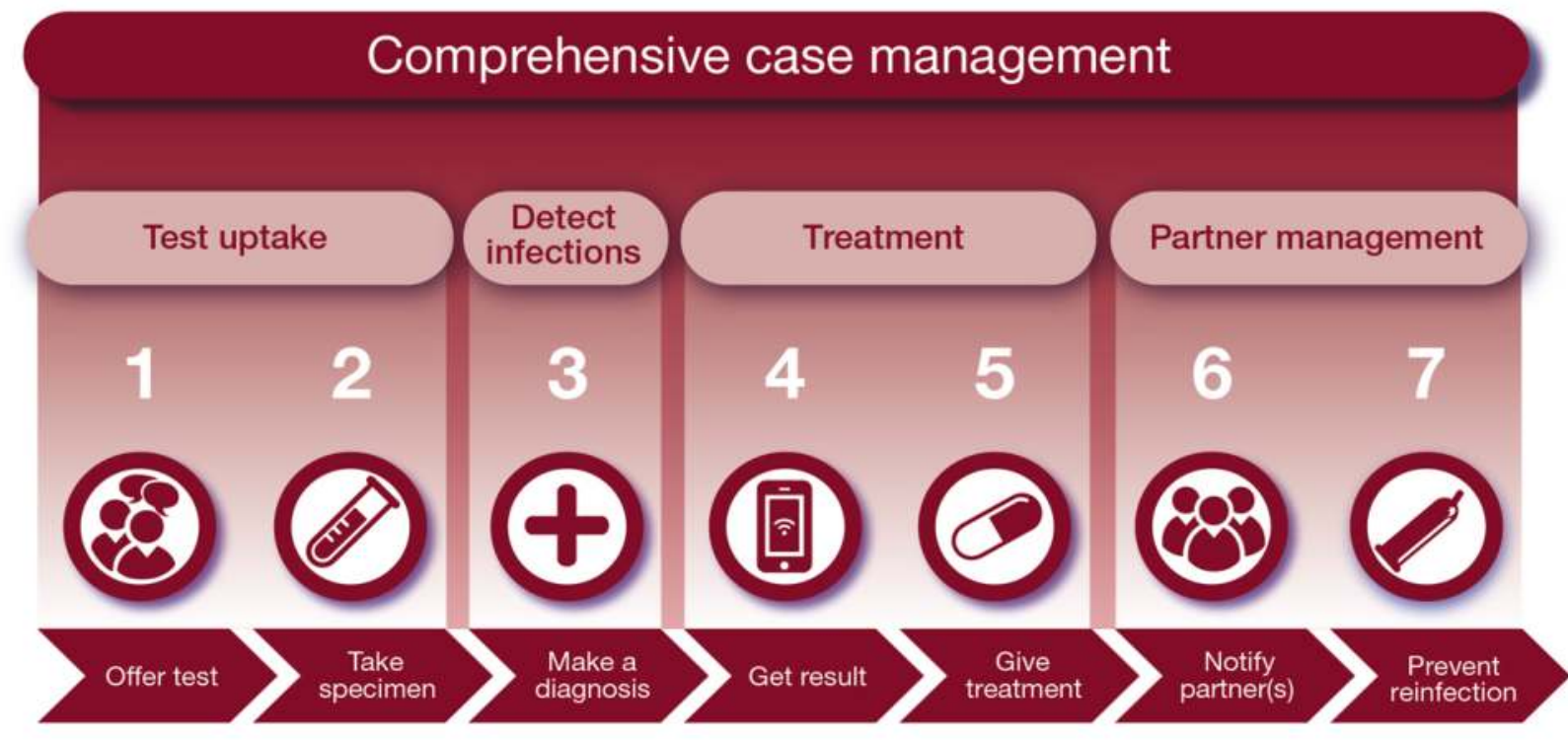

\section{Nottinghamshire Context:}

In 20 I4, Nottinghamshire achieved a chlamydia detection rate index (DRI) below regional and national index rates, whereas Nottingham City exceeded these rates.

In December 2015, the local Sexual Health Strategic Advisory Group (SHSAG) harnessed the opportunity to pilot the NCSP CCP Tool as a system enabler to look at the seven stages of screening to optimise local outcomes.

Figure 2: Chlamydia Detection Rates for England and East Midlands, 2014 Chlamydia detection rate / 100,000 aged 15-24 (PHOF indicator 3.02) 2014

\begin{tabular}{|c|c|c|c|c|}
\hline $\begin{array}{l}\text { Area } \\
\Delta \nabla\end{array}$ & $\begin{array}{l}\text { Count } \\
\Delta \nabla\end{array}$ & $\begin{array}{l}\text { Value } \\
\Delta \nabla\end{array}$ & $\begin{array}{c}95 \% \\
\text { Lower Cl }\end{array}$ & $\begin{array}{c}95 \% \\
\text { Upper Cl }\end{array}$ \\
\hline England & 137,993 & 2,012 & 2,001 & 2,023 \\
\hline East Midlands region & 12,418 & 2,050 & 2,014 & 2,086 \\
\hline Derby & 730 & 2,011 & 1,867 & 2,162 \\
\hline Derbyshire & 1,852 & 2,096 & 2,002 & 2,194 \\
\hline Leicester & 1,048 & 1,757 & 1,652 & 1,867 \\
\hline Leicestershire & 1,373 & 1,616 & 1,532 & 1,704 \\
\hline Lincolnshire & 1,750 & 2,045 & 1,951 & 2,144 \\
\hline Northamptonshire & 1,816 & 2,224 & 2,123 & 2,329 \\
\hline Nottingham & 2,026 & 2,807 & 2,686 & 2,932 \\
\hline Nottinghamshire & 1,757 & $1,900 \square$ & 1,812 & 1,991 \\
\hline Rutland & 66 & 1,390 & 1,075 & 1,769 \\
\hline
\end{tabular}

\section{Methods}

Routine surveillance data from GUMCAD and CTAD was used to populate the CCP for the area. Findings were discussed at the SHSAG and actions agreed.

\section{Findings and Actions}

Key issues identified were:

I Unknown test offer-rate (CCP Stage I)

2 Low coverage in Nottinghamshire County districts (CCP Stage I)

3 Low re-testing rates following treatment (CCP Stage 7)

In response:

I Existing GUMCAD codes were used to infer the offer of a test and identify where the offer of the test could be enhanced

2 The CCP informed commissioner priorities for a similar service offer across the city and county

3 New investment for online screening considered

4 A targeted approach was developed for health promotion to raise awareness of testing among key populations, primary care and providers of other young person services

5 An audit of re-testing pathways and a text reminder system for re-screening at 3 months was implemented in one of the units

\section{Conclusion}

The CCP approach:

- provided a strategic focus to increase multi-agency partner understanding of screening at all stages of the pathway

- confirmed the need for an integrated screening offer across testing providers

- confirmed the need to prioritise funding to commission an online Chlamydia Testing Service in the county

- enabled SHSAG to focus on areas in the pathway that had the potential to yield 'quick wins' (for example: offer of re-test)

The CCP approach and actions taken provided assurance to the Health and Wellbeing Boards of the targeted approach to drive forward and monitor improvements in local outcomes.

It is envisaged that an upward trend in the DRI will be seen in the medium term, the data will be reviewed in 2018 using the CCP tool.

\section{References}

I. PHE Towards achieving the chlamydia detection rate. Considerations for commissioning. 20I4. 2 PHE NCSP Chlamydia Care Pathway. PHE publications gateway number: 20I6307. Published May 20I6. 